\title{
Melhora da dor radicular após discectomia lombar transforaminal endoscópica em discos com alterações degenerativas avançadas
}

\section{Improvement in Radicular Pain after Endoscopic Transforaminal Lumbar Discectomy at Discs with Advanced Degenerative Changes}

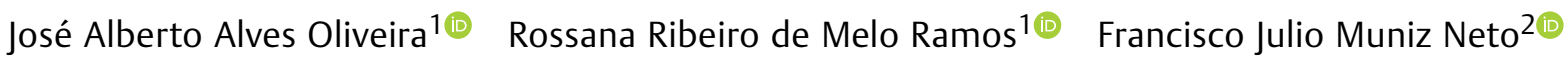
Paulo Cesar de Almeida ${ }^{3(\infty)}$ Max Rogerio Freitas Ramos ${ }^{1}{ }^{\circledR}$ Paulo Sergio Teixeira de Carvalho ${ }^{10}$

${ }^{1}$ Escola de Medicina, Universidade Federal do Estado do Rio de Janeiro, Rio de Janeiro, RJ, Brasil

${ }^{2}$ Serviço de Radiologia, Hospital Israelita Albert Einstein, São Paulo, SP, Brasil

${ }^{3}$ Centro de Ciências da Saúde, Universidade Estadual do Ceará Fortaleza, CE, Brasil

Rev Bras Ortop 2022;57(1):55-60.
Endereço para correspondência José Alberto Alves Oliveira, MD, Rua Bruno Porto, 600, Flat 2201-A, Parque Iracema, Fortaleza, Ceará, 60824-010, Brasil (e-mail: bertoalvesoliveira@gmail.com).

\section{Resumo \\ Palavras-chave \\ - ciática \\ - deslocamento do disco intervertebral \\ - região lombossacral}

Objetivos Avaliar a correlação entre as alterações radiológicas (Pfirrmann e Modic) e a intensidade da dor radicular em pacientes submetidos a cirurgia endoscópica transforaminal para hérnia de disco lombar.

Métodos Uma sequência de casos com 39 pacientes, 50 discos intervertebrais em avaliação pré-operatória, no período de 29 de janeiro de 2018 a 28 de agosto de 2019 , no serviço de cirurgia endoscópica da coluna vertebral. Os dados demográficos, indicação cirúrgica, detalhes operatórios e complicações foram todos obtidos junto aos prontuários clínicos. Os pacientes foram divididos em três grupos, com base na classificação Modic (ausência de Modic, Modic 1 e Modic 2) e em dois grupos, considerando a classificação de Pfirrmann (Pfirrmann IV e Pfirrmann V). Os dados foram processados no software IBM SPSS Statistics for Windows, versão 22.0 (IBM Corp., Armonk, NY, EUA), com nível de significância de $p<0,05$.

Resultados Não houve diferença entre os gêneros; idade: 50,36 $\pm 15,05$ anos; nível da doença: L2-L3 1 (2\%), L3-L4 2 (4\%), L4-L5 9 (18\%), L5-S1 8 (16\%), L3-L4 + L4-L5 4
O presente estudo foi desenvolvido no Curso de Cirurgia Minimamente Invasiva da Coluna da Universidade Federal do Estado do Rio de Janeiro, Rio de Janeiro, Brasil. recebido

12 de Setembro de 2020

aceito

11 de Fevereiro de 2021

Publicado on-line

Outubro 13, 2021
DOI https://doi.org/

$10.1055 / \mathrm{s}-0041-1732387$. ISSN 0102-3616. (c) 2021. Sociedade Brasileira de Ortopedia e Traumatologia. All rights reserved.

This is an open access article published by Thieme under the terms of the Creative Commons Attribution-NonDerivative-NonCommercial-License, permitting copying and reproduction so long as the original work is given appropriate credit. Contents may not be used for commercial purposes, or adapted, remixed, transformed or built upon. (https://creativecommons.org/ licenses/by-nc-nd/4.0/)

Thieme Revinter Publicações Ltda., Rua do Matoso 170, Rio de Janeiro, RJ, CEP 20270-135, Brazil 


\section{Abstract}

Keywords

- sciatica

- intervertebral disc displacement

- lumbosacral region
(8\%), e L4-L5 + L5-S1 26 (52\%); localização: foraminal direito em 7 pacientes (14\%), foraminal esquerdo em 15 pacientes (30\%), central em 9 pacientes (18\%), e difuso em 19 pacientes (38\%); dor radicular: esquerda em 25 pacientes (50\%), direita em 11 pacientes (22\%), e bilateral em 14 pacientes (28\%); escala visual analógica (EVA) préoperatório: 9,5 $\pm 0,91$, pós-operatório: $2,5 \pm 1,79$; tempo cirúrgico: $100 \pm 31,36$ minutos; e acompanhamento de $8,4 \pm 6,7$ meses. Foi registrada menos dor ciática pósoperatória nos grupos Modic 2 versus Modic $1(p<0,05)$. Não houve diferença na dor radicular pós-operatória entre os grupos Pfirrmann (IV versus $\mathrm{V}$ ).

Conclusão Embora não exista diferença clínica entre os grupos, em estágios avançados da degeneração discal, a discectomia transforaminal endoscópica mostrou-se eficaz na redução da dor radicular em pacientes com hérnia de disco lombar.

Objectives To evaluate the correlation between radiologic changes (Pfirrmann and Modic) and radicular pain intensity in patients who underwent transforaminal endoscopic surgery for lumbar disc herniation.

Methods Series of cases with 39 patients, 50 intervertebral discs in preoperative evaluation from January 29, 2018 to August 28, 2019 in an endoscopic spine surgery service. Demographic data, surgical indication, operative details and complications were obtained from medical records. The patients were divided into three groups based on the Modic classification (Modic absence, Modic 1 and Modic 2) and into two groups considering the Pfirrmann classification (Pfirrmann IV and Pfirrmann V). Data were processed in IBM SPSS Statistics for Windows, Version 22.0 (IBM Corp., Armonk, NY, USA), with a significance level of $p<0,05$.

Results There was no difference between genders; age: $50,36 \pm 15,05$ years old; disease level: L2-L3 1 (2\%), L3-L4 2 (4\%), L4-L5 9 (18\%), L5-S1 8 (16\%), L3-L4 + L4-L5 4 (8\%), and L4-L5 + L5-S1 26 (52\%); location: right foraminal 7 (14\%), left foraminal 15 (30\%), central 9 (18\%) and diffuse 19 (38\%); radicular pain: left 25 (50\%), right 11 (22\%), and bilateral 14 (28\%); preoperative visual analogue scale (VAS): $9,5 \pm 0,91$, postoperative: $2,5 \pm 1,79$; surgery duration: $100 \pm 31,36$ minutes; and follow-up: $8,4 \pm 6,7$ months. Less postoperative sciatica was registered in the Modic 2 versus Modic 1 group $(p<0,05)$. There was no difference in the postoperative radicular pain between the Pfirrmann groups (IV versus $V$ ).

Conclusion Although there is no clinical difference between the groups, in advanced stages of disc degeneration, endoscopic transforaminal discectomy proved to be effective in diminishing radicular pain in patients with lumbar disc herniation.

\section{Introdução}

A microdiscectomia aberta é o tratamento padrão ouro para hérnia de disco lombar. No entanto, a Discectomia lombar endoscópica percutânea apresenta muitos benefícios em comparação com a cirurgia aberta, tais como: menor trauma cirúrgico, mínima perda sanguínea, menor tempo de internação, recuperação mais rápida e menor morbidade pósoperatória, em virtude da preservação da musculatura dorsal e das estruturas osteoligamentares. Este pequeno trauma tissular durante a cirurgia endoscópica resulta em uma reabilitação mais rápida, levando a menores custos para a sociedade. $^{1-5}$

O tratamento da hérnia de disco lombar com discopatia avançada, representada por estágios mais avançados na classificação de Modic e Pfirrmann, ainda não tem uma definição entre a discectomia ou artrodese.

Diante disso, o objetivo do presente estudo é avaliar a correlação entre as alterações radiológicas (Pfirrmann e Modic) e a variação de intensidade da dor radicular (pré- e pós-operatória), em pacientes submetidos a cirurgia endoscópica transforaminal para hérnia de disco lombar.

\section{Materiais e Método}

Uma série de casos com uma amostra inicial de 80 pacientes submetidos a tratamento cirúrgico para hérnia de disco lombar por via endoscópica transforaminal percutânea entre 29 de janeiro de 2018 (primeira entrada deste procedimento no banco de dados) e 28 de agosto de 2019 (último paciente com 
acompanhamento mínimo de 3 meses) no serviço de cirurgia endoscópica da coluna.

Os critérios de inclusão foram: dor radicular, falha do tratamento conservador de 12 semanas e diagnóstico de hérnia de disco lombar por meio de ressonância magnética (RM). Os critérios de exclusão foram: outra causa de dor que não a hérnia de disco lombar, artrodese lombar prévia, espondilolistese, tumor, infecção, fratura lombar e os graus de Pfirrmann I, II e III. Nos pacientes que apresentavam dor lombar e ciática, foram incluídos apenas aqueles cuja fonte da dor era principalmente radicular. Devido à pequena amostra e à incapacidade de análise da associação, foram excluídos os pacientes com grau III de Pfirrmann.

Os pacientes selecionados foram investigados quanto aos dados demográficos, às indicações cirúrgicas e aos detalhes cirúrgicos registrados nos prontuários, assim como ao registro pré- e pós-operatório da escala visual analógica (EVA) (variando de 0 a 10 , sendo 0 sem dor e 10 a dor mais intensa já experimentada na perna), onde a melhora em 2 pontos foi considerada como um bom resultado clínico.,

Os parâmetros radiológicos (classificações de Pfirrmann e Modic) foram realizados por um radiologista experiente especializado em doenças da coluna vertebral, sem acesso às características clínicas do paciente.

Os pacientes foram divididos em três grupos de acordo com a classificação Modic (ausência de Modic, Modic 1 e Modic 2) e em dois grupos de acordo com a classificação de Pfirrmann (Pfirrmann IV e Pfirrmann V).

Todos os procedimentos foram realizados por um único cirurgião (Carvalho P.S.T.), com experiência de $\sim 30$ anos em cirurgia endoscópica espinhal. Os pacientes receberam anestesia local, sendo sedados com propofol e remifentanila. Os pacientes foram posicionados em decúbito ventral em uma mesa radiotransparente sob controle fluoroscópico ortogonal de dois planos, sobre um suporte para o quadril e tórax, a fim de aliviar os órgãos abdominais e torácicos e diminuir a hemorragia epidural. A mesa cirúrgica pôde ser ajustada para cifose intraoperatória no nível lombar. O cirurgião operou do lado do prolapso discal, sendo que o monitor de vídeo e o braço em $\mathrm{C}$ foram posicionados no lado oposto.

Após a determinação da posição da crista ilíaca, a incisão na pele tinha uma distância desde a linha média até o ponto de punção, conforme o nível abordado (6 a $8 \mathrm{~cm}$ para L2-L3, 8 a $10 \mathrm{~cm}$ para L3-L4 e 12 a $14 \mathrm{~cm}$ para L4-5 e L5-S1). Após a assepsia, o ponto de entrada foi marcado, sempre superior à crista ilíaca, sendo traçada uma linha através do processo articular superior (PAS) até a linha média da placa terminal inferior.

A agulha espinhal é inserida ortogonalmente ao espaço discal. O ideal é que, quando observada por meio do fluoroscópio, a ponta da agulha avance até a linha posterior do corpo vertebral na incidência lateral e até o meio da linha do pedículo medial na incidência anteroposterior. Com a agulha espinhal alojada no disco, o núcleo pulposo foi corado em azul (usando uma adsorção de $2 \mathrm{~mL}$ como meio de contraste e o corante azul de metileno para discografia), prosseguindo para as etapas seguintes: passagem do fio-guia através da agulha espinhal, remoção da agulha espinhal, incisão limitada $(8 \mathrm{~mm})$ na pele do local de entrada, distribuição do obturador canulado cônico ao longo do fio-guia, inserção do obturador no disco (ao atingir o anel), avanço da cânula de trabalho de formato oval e chanfrada (para dentro do disco) ao longo do obturador e a remoção do obturador. Em seguida, o endoscópio é inserido através da cânula, e o núcleo patológico (corado em azul para facilitar a distinção e preso à fissura anular) e qualquer tecido fibroso cicatricial são liberados, sendo completamente removidos com o uso de um fórceps endoscópico e de um dispositivo de radiofrequência. A cânula de trabalho é ajustada para localizar e remover a faceta superior hiperplásica, o disco herniado, a borda posterior do corpo vertebral e os osteófitos que existem ao redor da raiz transversal, com a utilização de uma broca de alta velocidade, escareador ou cortador ósseo (de dentro para fora).

Para que a descompressão seja considerada satisfatória, é necessário que o nervo apresente pulsações semelhantes à frequência cardíaca e que a quantidade de material removido do disco seja equivalente à quantidade observada na RM. Ao completar o procedimento, o endoscópio é retirado e a pele é suturada.

Os dados foram processados no programa IBM SPSS Statistics for Windows, Versão22.0 (IBM Corp., Armonk, NY, EUA), licença US \# 10101131007, onde foram calculados as médias e o desvio padrão (DP). A comparação das variáveis do grupo Modic foi realizada mediante o teste da razão de verossimilhança e o teste de Kruskal-Wallis. O grupo Pfirrmann utilizou o teste da razão de verossimilhança, o teste $\mathrm{t}$ de Student para dados pareados e o teste de Mann-Whitney. $\mathrm{O}$ nível de significância considerado foi $p<0,005$.

O estudo foi aceito pelo Comitê de Ética e Pesquisa sob o número de aceitação 4.191.443.

\section{Resultados}

A partir de uma amostra inicial com 80 pacientes, foram selecionados 39, sendo avaliados 50 discos intervertebrais. Não houve diferença entre o gênero dos pacientes; idade: 50,36 \pm 15,05 anos; nível de cirurgia: L2-L3 1 (2\%), L3-L4 2 (4\%), L4-L5 9 (18\%), L5-S1 8 (16\%), L3-L4 + L4-L5 4 (8\%), e L4-L5 + L5-S1 26 (52\%); localização da hérnia: foraminal direito 7 (14\%), foraminal esquerdo 15 (30\%), central 9 (18\%) e difuso 19 (38\%); dor nas pernas: esquerda 25 (50\%), direita 11 (22\%), e dois lados 14 (28\%); EVA: pré-operatório $9,5 \pm 0,91$, pós-operatório $2,5 \pm 1,79$; tempo cirúrgico $100 \pm 31,36$ minutos, e acompanhamento de 8,4 $\pm 6,7$ meses.

Ao comparar os grupos com as alterações Modic, notou-se que houve menor intensidade de dor radicular no pósoperatório do grupo Modic2 em comparação com o grupo Modic 1 (-Tabela 1).

Ao analisar os grupos Pfirrmann (Pfirrmann IV versus Pfirrmann V), não houve diferença na comparação das características clínicas e radiológicas, tampouco na intensidade da dor ciática (- Tabela $\mathbf{2}$ ).

\section{Discussão}

Em um estudo de meta-análise com nove ensaios clínicos randomizados, ao comparar a cirurgia endoscópica com a 
Tabela 1 Características clínicas e radiológicas entre os grupos MODIC

\begin{tabular}{|c|c|c|c|c|}
\hline & AUSÊNCIA DE MODIC & MODIC 1 & MODIC 2 & valor-p \\
\hline \multicolumn{5}{|l|}{ Gênero } \\
\hline Masculino & $11 / 46 \%$ & $6 / 25 \%$ & $7 / 29 \%$ & \multirow[t]{2}{*}{$0,751^{1}$} \\
\hline Feminino & $10 / 38 \%$ & $9 / 35 \%$ & $7 / 27 \%$ & \\
\hline Idade (Anos) & $50,14 \pm 13,58$ & $49,67 \pm 20,61$ & $46,64 \pm 13,90$ & $0,800^{2}$ \\
\hline \multicolumn{5}{|l|}{ Nível de Cirurgia } \\
\hline L5-S1 & $5 / 62 \%$ & $1 / 13 \%$ & $2 / 25 \%$ & \multirow[t]{6}{*}{$0,219^{1}$} \\
\hline L4-L5 & $3 / 33 \%$ & $1 / 11 \%$ & $5 / 56 \%$ & \\
\hline L3-L4 & $1 / 50 \%$ & $1 / 50 \%$ & 0 & \\
\hline L2-L3 & $1 / 100 \%$ & 0 & 0 & \\
\hline L4-L5 L5-S1 & $10 / 38 \%$ & $9 / 35 \%$ & $7 / 27 \%$ & \\
\hline L3-L4 L4-L5 & $1 / 25 \%$ & $3 / 75 \%$ & 0 & \\
\hline \multicolumn{5}{|l|}{ Localização da Hérnia } \\
\hline Foraminal Direito & $3 / 43 \%$ & $3 / 43 \%$ & $1 / 14 \%$ & \multirow[t]{4}{*}{$0,813^{1}$} \\
\hline Foraminal Esquerdo & $6 / 40 \%$ & $3 / 20 \%$ & $6 / 40 \%$ & \\
\hline Central & $3 / 33,3 \%$ & $3 / 33,3 \%$ & $3 / 33,3 \%$ & \\
\hline Difusa & $9 / 47 \%$ & $6 / 32 \%$ & $4 / 21 \%$ & \\
\hline \multicolumn{5}{|l|}{ Dor na Perna } \\
\hline Esquerda & $10 / 40 \%$ & $6 / 24 \%$ & $9 / 36 \%$ & \multirow[t]{3}{*}{$0,466^{1}$} \\
\hline Direita & $6 / 55 \%$ & $4 / 36 \%$ & $1 / 9 \%$ & \\
\hline Ambos os Lados & $5 / 36 \%$ & $5 / 36 \%$ & $4 / 28 \%$ & \\
\hline \multicolumn{5}{|c|}{ Dor na Perna Escala Visual Analógica } \\
\hline Pré-operatório & $9,71 \pm 0,78$ & $9,20 \pm 0,86$ & $9,64 \pm 0,92$ & $0,872^{2}$ \\
\hline Pós-operatório & $2,33 \pm 1,68$ & $2,93 \pm 2,25$ & $1,93 \pm 1,14$ & $p<0,0001^{2}$ \\
\hline$\Delta$ (Pós-Pré) & $-7,38 \pm 1,77$ & $-6,26 \pm 2,31$ & $-7,71 \pm 1,54$ & $0,183^{2}$ \\
\hline Acompanhamento (meses) & $11,25 \pm 7,33$ & $7,65 \pm 7,89$ & $7,36 \pm 5,81$ & $0,132^{2}$ \\
\hline
\end{tabular}

1- Razão de verossimilhança; 2- Kruskal -Wallis.

abordagem aberta para hérnia de disco lombar sintomática, verificou que a satisfação do paciente foi maior e o tempo de hospitalização, menor, no grupo que foi submetido à cirurgia endoscópica. $^{8}$

Ao considerar a anatomia da coluna lombar, onde as dimensões do forame intervertebral diminuem à medida que os espaços interlaminares aumentam de L1 para L5, a abordagem transforaminal é recomendada para níveis lombares elevados incluindo L3/L4 e para os casos que apresentam estenose foraminal ou do recesso lateral, A abordagem interlaminar é sugerida para os níveis L4/L5 e L5/S1, além de para os casos nos quais ocorra estenose do recesso central e lateral. $^{9}$

A vantagem mais significativa da abordagem transforaminal, em detrimento da interlaminar, seria o fato da primeira ser realizada sob anestesia local em ambiente ambulatorial, evitando o risco da anestesia geral (utilizada para a abordagem interlaminar), principalmente em pacientes idosos com comorbidades, resultando em menores custos com esse tipo de paciente para o sistema de saúde. ${ }^{10}$
Xu et al. ${ }^{11}$ estudaram a diferença no resultado clínico nos pacientes submetidos a cirurgia endoscópica transforaminal para hérnia de disco lombar, considerando as alterações Modic. Nos três grupos analisados (Controle, Modic tipo I e Modic tipo II), houve melhora significativa na intensidade da dor radicular, medida por meio da EVA, em 3 meses, 1 ano e no último ano de acompanhamento pós-operatório, em comparação com os valores pré-operatórios. É importante mencionar que não houve diferença entre os três grupos.

Esses autores encontraram a presença de lesão nas raízes nervosas em 5 pacientes: 3 no grupo controle, 1 no Modic tipo I e 1 no Modic tipo II, com taxas de complicações de 6,6, 6,8 e $8 \%$ nestes grupos, respectivamente. A recorrência ocorreu em 16 pacientes: 8 no grupo controle, 4 no grupo Modic tipo 1 e 4 no grupo Modic tipo II, com taxas de 4,4, 9,1 e $8 \%$ nestes três grupos, respectivamente. ${ }^{11}$

Em nosso estudo, foi observada uma melhora significativa na dor ciática pós-operatória nos três grupos analisados (ausência de Modic, Modic 1 e Modic 2), após a discectomia endoscópica transforaminal. No entanto, houve uma diferença estatisticamente significativa no valor da EVA pós- 
Tabela 2 Características clínicas e radiológicas entre os grupos PFIRRMANN

\begin{tabular}{|c|c|c|c|}
\hline & Pfirrmann iv & Pfirrmann v & valor-p \\
\hline \multicolumn{4}{|l|}{ Gênero } \\
\hline Masculino & $16 / 73 \%$ & $6 / 27 \%$ & \multirow[t]{2}{*}{$0,723^{1}$} \\
\hline Feminino & $17 / 68 \%$ & $8 / 32 \%$ & \\
\hline Idade (Anos) & $47,45 \pm 16,17$ & $53,29 \pm 16,95$ & $0,271^{2}$ \\
\hline \multicolumn{4}{|l|}{ Nível de Cirurgia } \\
\hline L5-S1 & $7 / 78 \%$ & $2 / 22 \%$ & \multirow[t]{6}{*}{$0,562^{1}$} \\
\hline L4-L5 & $7 / 78 \%$ & $2 / 22 \%$ & \\
\hline L3-L4 & $2 / 100 \%$ & 0 & \\
\hline L2-L3 & $1 / 100 \%$ & 0 & \\
\hline L4-L5 L5-S1 & $13 / 59 \%$ & $9 / 41 \%$ & \\
\hline L3-L4 L4-L5 & $3 / 75 \%$ & $1 / 25 \%$ & \\
\hline \multicolumn{4}{|l|}{ Localização da Hérnia } \\
\hline Foraminal Direito & $7 / 100 \%$ & 0 & \multirow[t]{4}{*}{$0,096^{1}$} \\
\hline Foraminal Esquerdo & $10 / 62 \%$ & $6 / 38 \%$ & \\
\hline Central & $7 / 78 \%$ & $2 / 22 \%$ & \\
\hline Difusa & $9 / 60 \%$ & $6 / 40 \%$ & \\
\hline \multicolumn{4}{|l|}{ Dor na Perna } \\
\hline Esquerda & $16 / 62 \%$ & $10 / 38 \%$ & \multirow[t]{3}{*}{$0,201^{1}$} \\
\hline Direita & $9 / 90 \%$ & $1 / 10 \%$ & \\
\hline Ambos os Lados & $8 / 73 \%$ & $3 / 27 \%$ & \\
\hline Pré-operatório & $9,42 \pm 0,97$ & $9,29 \pm 0,, 91$ & $0,539^{3}$ \\
\hline Pós-operatório & $2,42 \pm 1,56$ & $2,43 \pm 2,20$ & $0,737^{3}$ \\
\hline$\Delta$ (Pós-Pré) & $-7,00 \pm 1,83$ & $-6,85 \pm 2,17$ & $0,902^{3}$ \\
\hline Acompanhamento (meses) & $8,57 \pm 7,30$ & $7,77 \pm 6,90$ & $0,822^{3}$ \\
\hline
\end{tabular}

1- Razão de verossimilhança; 2- T de Student; 3- Mann-Whitney.

operatória entre os grupos Modic 1 e Modic 2, sendo de maior intensidade no último grupo. A diferença não foi considerada clinicamente significativa porque não era $>2$ pontos na EVA.

A presente pesquisa evidenciou a presença de paresia (força muscular grau IV na escala do Medical Research Council) em seis pacientes no pré-operatório: dois no grupo ausência de Modic, um no grupo Modic 2, e três em pacientes não classificados. Um paciente do grupo Modic 1 apresentou paresia após a cirurgia. Esses pacientes foram submetidos a reabilitação motora pós-operatória, sendo que a fraqueza muscular foi normalizada após $\sim 3$ meses de tratamento. A recidiva foi observada em cinco pacientes, sendo três no grupo ausência de Modic, um no grupo Modic 1, e um em um paciente não classificado.

Considerando as complicações, um estudo multicêntrico com $>26.000$ casos encontrou prevalência $<1 \%$ para discectomia endoscópica percutânea, tais como: disestesia $0,45 \%$, rupturas durais $0,17 \%$, discite $0,25 \%$, deficiência sensitivomotora $0,32 \%$, e recorrência $0,79 \%^{12}$

Os fatores de risco gerais para a recorrência em cirurgia endoscópica percutânea são: sexo masculino, obesidade, idade $>50$ anos, história de trauma, e hérnia de disco central.
Porém, existem fatores relacionados à técnica cirúrgica, tais como: cirurgiões menos experientes ( $<200$ casos) e uso de material inadequado. ${ }^{9,13}$

Em nosso estudo, todos os pacientes foram submetidos à técnica transforaminal para hérnia de disco lombar em discos com degeneração avançada (Pfirrmann IV e V). Foi observada uma melhora significativa, não só estatisticamente, mas também clinicamente, na intensidade da dor ciática, de acordo com a EVA no último acompanhamento pós-operatório; contudo, não houve diferença entre os dois grupos.

Na presente pesquisa, a cirurgia endoscópica transforaminal para hérnia de disco lombar com discopatia avançada representada por estágios mais avançados nas classificações de Modic e Pfirrmann demonstrou ser clinicamente eficaz na redução da dor (pré-operatório forte a pós-operatório leve). Não houve diferença clínica para aqueles pacientes que apresentavam doença degenerativa discal avançada.

As limitações da presente pesquisa foram o tamanho da amostra, o acompanhamento relativamente curto dos pacientes, realizado em apenas um centro especializado; no entanto, a presente pesquisa ainda traz dados importantes sobre a doença degenerativa discal avançada tratada com método minimamente invasivo. 
60 Melhora da dor radicular após discectomia lombar transforaminal endoscópica Oliveira et al.

\section{Conclusão}

O presente estudo mostrou que, mesmo na degeneração discal avançada, a discectomia endoscópica percutânea transforaminal parece ser um método eficiente na redução da dor radicular em pacientes com hérnia de disco lombar.

A melhora da dor pós-operatória foi observada em todos os grupos analisados pela classificação Modic (ausência de Modic, Modic 1 e Modic 2) ou pela classificação de Pfirrmann (Pfirrmann IV e Pfirrmann V), sem diferença clínica na redução da dor ciática entre os grupos.

\section{Suporte Financeiro}

Não houve suporte financeiro de fontes públicas, comerciais, ou sem fins lucrativos.

\section{Conflito de Interesses}

Os autores declaram não haver conflitos de interesses.

\section{Referências}

1 Kapetanakis S, Gkasdaris G, Thomaidis T, Charitoudis G, Kazakos K. Comparison of quality of life between men and women who underwent Transforaminal Percutaneous Endoscopic Discectomy for lumbar disc herniation. Int J Spine Surg 2017;11(04):28-40

2 Gadjradj PS, van Tulder MW, Dirven CM, Peul WC, Harhangi BS. Clinical outcomes after percutaneous transforaminal endoscopic discectomy for lumbar disc herniation: a prospective case series. Neurosurg Focus 2016;40(02):E3-E9

$3 \mathrm{Wu}$ J, Zhang C, Zheng W, Hong CS, Li C, Zhou Y. Analysis of the Characteristics and Clinical Outcomes of Percutaneous Endoscopic Lumbar Discectomy for Upper Lumbar Disc Herniation. World Neurosurg 2016;92:142-147
4 Jiang X, Zhou X, Xu N. Clinical effects of transforaminal and interlaminar percutaneous endoscopic discectomy for lumbar disc herniation: A retrospective study. Medicine (Baltimore) 2018;97(48):e13417-e13422

5 Li Y, Wang B, Wang S, Li P, Jiang B. Full-Endoscopic Decompression for Lumbar Lateral Recess Stenosis via an Interlaminar Approach versus a Transforaminal Approach. World Neurosurg 2019;128: e632-e638

6 Falavigna A, Teles AR, Braga GL, Barazzetti DO, Lazzaretti L, Tregnago AC. Instrumentos de Avaliação Clínica e Funcional em Cirurgia da Coluna Vertebral. Coluna/Columna 2011;10(01): 62-67

7 Oliveira JAA, Guerra e Silva VT, Almeida, LEPCAd, Meireles RdSP, Lopes GdR, Mello APd, Almeida PC, Silva LECTd. T1 pelvic angle in improvement of pain in high-grade lumbar spondylolisthesis. Coluna/Columna 2020;19(02):108-111

8 Cong L, Zhu Y, Tu G. A meta-analysis of endoscopic discectomy versus open discectomy for symptomatic lumbar disk herniation. Eur Spine J 2016;25(01):134-143

9 Pan M, Li Q, Li S, et al. Percutaneous Endoscopic Lumbar Discectomy: Indications and Complications. Pain Physician 2020;23 (01):49-56

10 Ruetten S, Komp M. Endoscopic Lumbar Decompression. Neurosurg Clin N Am 2020;31(01):25-32

$11 \mathrm{Xu} \mathrm{J}$, Li Y, Wang B, et al. Percutaneous Endoscopic Lumbar Discectomy for Lumbar Disc Herniation with Modic Changes via a Transforaminal Approach: A Retrospective Study. Pain Physician 2019;22(06):E601-E608

12 Zhou C, Zhang G, Panchal RR, et al. Unique Complications of Percutaneous Endoscopic Lumbar Discectomy and Percutaneous Endoscopic Interlaminar Discectomy. Pain Physician 2018;21 (02):E105-E112

13 Park CH, Park ES, Lee SH, et al. Risk Factors for Early Recurrence After Transforaminal Endoscopic Lumbar Disc Decompression. Pain Physician 2019;22(02):E133-E138 